\title{
MÉTODO DO PERFIL CULTURAL PARA AVALIAÇÃO DO ESTADO FÍSICO DE SOLOS EM CONDIÇÕES TROPICAIS ${ }^{(1)}$
}

\author{
J . TAVARES FILHO(2), R. RALISCH ${ }^{(2)}$, M. F. GUIMARÃE $S^{(2)}$, C. C . \\ MEDINA ${ }^{(2)}$, L. C. BALBINO(3) \& C. S. V.J . NEVES ${ }^{(2)}$
}

\begin{abstract}
RESUMO
Uma nova abordagem para adoção do método do Perfil Cultural em condições de solos tropicais é aqui tratada. Analisou-se a evolução desse método desde seu início na França, na década de 1960, até a proposta de sua utilização no estudo de manejo de solos tropicais. Tal método mostra-se eficiente no diagnóstico quali tativo do estado físi co dos solos no campo, na orientação de amostragem de solos, no estudo dos efeitos da antropização, na visualização das interações físicas, químicas e biológicas dos solos, nos estudos de mecânica do solo no campo e no estudo da organização estrutural de solos agrícolas, além de auxiliar na análise do sistema radicular.
\end{abstract}

Termos de indexação: manejo do solo, estrutura do solo, diagnóstico do manejo

\section{SUMMARY: CULTURAL PROFILE METHODOLOGY FOR SOIL PHYSICAL EVALUATION UNDER TROPICAL CONDITIONS}

A new approach is discussed for adoption of criteria in the application of the cultural profilemethodol ogy for tropical soil conditions. The evol ution of this methodol ogy has been analyzed since its beginning in France in the 1960s, until its proposal for tropical soil management studies. Thecultural profileapproach can beused to makequal itativediagnosis of the physical state of soil in the field, as well as to help with soil sampling, to study the effect of agricultural machinery, to visualize the physical, chemical and biological soil interactions, and to help with thestudies of contraction, expansion, and shearing in thefield and in the structural organization of soils and root system analyses.

Index terms: soil physics, soil management, soil structure

\footnotetext{
(1) Recebido para publicação em junho de 1996 e aprovado em dezembro de 1998.

(2) Departamento de Agronomia, CCA, Universidade Estadual de Londrina. Caixa Postal 6001, CEP 86051-990 Londrina (PR). E-mail: tavares@mpd.uel.br

(3) Pesquisador da Embrapa Arroz e Feijão. Caixa Postal 179, CEP 74001-970 Goiânia (GO).
} 


\section{NTRODUÇÃO}

A partir do uso da motomecanização na agricultura em meados do séculoXX na Europa, nova abordagem da avaliação da fertilidade do solo foi exigida, uma vez que os efeitos físicos assumiram grande importância em virtude da ação de máquinas e implementos. Portanto, não só a quantidade de elementos nutritivos passou a interessar, mas também as condições que as raízes encontravam, possibilitando o melhor aproveitamento desses elementos (Hénin et al., 1960).

Hoje, sabe-sequeo manejo adequado do solo, além da preservação desse recurso natural, é de fundamental importância na produção agrícola. Essa adequação depende dos objetivos da exploração, do sistema agrícola adotado, do ti po e das condições do sol o trabalhado.

Considerando que o manejo varia no tempo e no espaço e de região para região, a avaliação dos diferentes sistemas de manejo é extremamente complexa. Em virtude de tal complexidade, uma gama muito grande de análises é utilizada em termos quantitativos e, mesmo assim, raramente é representativa da realidade, dada a variabilidade espacial das características e propriedades físicas dos solos.

Segundo Manichon (1995), um procedimento ainda bastante utilizado é o estudo das correlações entre as técnicas culturais e os rendimentos das culturas. Espera-se da repetição (no tempo e no espaço) da comparação dos rendimentos obtidos, segundo di ferentes modal idades de manejo de sol os, o estabel ecimento derel ações estáveis, suficientemente gerais para serem extrapoláveis e utilizáveis como referência. Para Sebillote (1975) e Wingate-Hill (1978), citados por Manichon (1995), na maioria dos casos isto não ocorre no estudo do manejo físico dos solos. Além disso, a complexidade no estudo desses fenômenos e a obtenção de respostas consistentes a esses problemas são ainda mais difíceis em solos tropicais (Ralisch, 1995). De acordo com Letey (1985), é muito difícil estabelecer uma relação entre a produtividade das culturas eas características físicas do solo em função de suas variabilidades tanto no tempo quanto na posição dentro do perfil do solo.

Assim, segundo Guimarães et al. (1993), no diagnóstico das condições físicas do solo, devem ser utilizados métodos que permitam avaliar o maior número possível de interações existentes e determinar quais são as ações e as formas de exploração mais adequadas para cada solo. Deve-se, então, adotar uma nova atitude deanálise que, para Manichon (1995), consisteem:

- melhor definir as variáveis explicativas, pois, sem se observar diretamenteo solo, as conclusões a respeito da ação dos implementos sobre el e serão resultado de suposição e não verificações concretas; e
- considerar o "estado do sol o" no estudo das rel ações trabalho do solo - rendimento das culturas.

Depara-se aqui com o conceito do Perfil Cultural (Hénin et al., 1960; Hénin et al., 1969), que permite uma nova visão de análise dos problemas agronômicos e, em particular, de análise dos efeitos da exploração agropecuária do solo. É realizado por meio da abertura de trincheiras e da observação da organização e da morfologia das estruturas do solo nas diferentes regi ões do perfil, que, segundoTavares Filho (1995), são conseqüência direta do estado de evolução pedológica de cada solo combinado com a ação antrópica que influi nessa evolução.

O Perfil Cultural foi inicialmente utilizado como uma ferramenta pedagógica na formação de estudantes de Agronomia. A partir da década de 70, Charreau \& Nicou (1971) e Gras et al. (1971) passaram a utilizar tal método como uma variável explicativa dos itinerários experimentais e, segundo Manichon \& Bodet (1976), citados por Manichon (1995), foram feitas tentativas no I nstituto Técnico de Cereais e Forrageiras (França) para tornar o procedimento de exame do perfil cultural mais operacional, mas sem resultados satisfatórios. $\mathrm{O}$ prosseguimento das refl exões levou à adoção de uma atitude claramente determinista:

- primeiramente, na definição de critérios de caracterização dos estados de horizontes antropizados, pela definição de dois níveis de organização estrutural, isto é, do estado interno dos torrões e do seu modo de organização, sendo as formas de evolução de um estado a outro, em cada nível de organização, elucidadas (Manichon, 1982); e

- em seguida, na forma de aplicação desses critérios ao Perfil Cultural. As interações de limites horizontais e verticais definem compartimentos que sofreram ações mecânicas definidas (Manichon, 1982; Manichon, 1988; Manichon \& Roger Estrada, 1990);

Essa dupla estratificação fornece, então, uma geografia dos horizontes antropizados que, segundo Manichon (1995), revela-se eficaz:

- como meio de análise e de diagnóstico da origem do estado observado (confrontação com dados históricos), utilizando critérios morfológicos previamentedefinidos (Ralisch et al. 1995; Tavares Filho, 1995);

- como plano de sondagem para a escol ha dos locais de coleta de amostras para análises físicas, químicas e biológicas (Papy, 1986; Medina, 1993; Tavares Filho, 1995); e

- como meio para auxílio no estudo do sistema radicular (Tardieu \& Manichon, 1986; Tardieu, 1988; Tavares Filho et al., 1991).

Objetiva-se, nestetrabal ho, mostrar a adequação desse método às condições de sol os tropicais ealguns resultados de sua aplicação em Latossolos do Paraná. 


\section{A METODOLOGIA}

Para utilização do Perfil Cultural, as trincheiras podem ser abertas tanto perpendicularmente como longitudinalmente ao sentido de trabalho do solo pel os implementos, segundo o objetivo da avaliação, com vistas a detectar as modificações estruturais provocadas no solo. A terra deve ser retirada de forma que se preservem totalmente a superfície e as laterais dos perfis a serem avaliados.

O método é fundamentado no estudo da morfologia do solo e consiste na delimitação dos volumes antropizados distintos, tanto em profundidade comolateralmente, a partir de critérios como: forma, tamanho e distribuição dos el ementos estruturais; presença ou ausência de poros visíveis a ol ho nu e continuidade destes; forma e dureza de agregados e torrões, dentre outros fatores.

Os critérios para descrição do modo de organização do perfil e do estado interno dos torrões constam nos quadros 1 e 2 .

Deve-se ressaltar que, por ter sido este método desenvol vido para as condições de sol os temperados, algumas dificuldades foram encontradas para sua aplicação em condições de solos tropicais. A observação detalhada da organização estrutural, conforme a proposta de Gautronneau \& Manichon (1987), mostrou-se rigorosa demais no estudo, por exemplo, dos efeitos da mecanização sobre o solo, dificultando a sua interpretação.

Dessa forma, buscou-se a utilização de critérios mais simplificados, mais abrangentes em sua interpretação, privilegiando-se mais o modo de organização do que o estado interno dos torrões. Obteve-se, assim, um método de diagnóstico particularmente adaptado ao estudo da evol ução da estrutura dos solos cultivados e dos diferentes sistemas de manejo em meio tropical.

Nessa adequação, procurou-se engl obar todas as variáveis relatadas até o presente momento, de forma mais simplificada, mas ao mesmo tempo mais descritiva. Trata-se de uma classificação piramidal em dois níveis: numa primeira etapa, estuda-se o modo de organização do perfil considerado (Quadro 1), diferenciando-se os volumes de solo visual mente alterados pel o manejo agrícola (AM) do visualmente não alterado pelo manejo agrícola

\section{Quadro 1. Modos de organização do perfil de solo(1)}

\begin{tabular}{|c|c|c|}
\hline Nível de análise & Simbologia & Definição \\
\hline \multirow[t]{2}{*}{ I } & AM & $\begin{array}{l}\text { Volume de solo visualmente alterado pelo manejo, isto é, pelo maquinário } \\
\text { agrícola e pelas raízes. }\end{array}$ \\
\hline & NAM & Volume de solo visualmente não alterado pelo manejo. \\
\hline 11 & L & $\begin{array}{l}\text { Volume de solo livre, solto, constituído por terra fina, solo pulverizado, } \\
\text { agregados e torrões de tamanhos variados (de } 0 \text { a } 10 \mathrm{~cm} \text { ) sem nenhuma coesão. } \\
\text { Comum na superfície dos solos trabalhados. Pode apresentar raízes em grandes } \\
\text { quantidades, bem ramificadas, não achatadas e não tortuosas, orientadas em } \\
\text { todas as direções. A estabilidade em água e a coesão a seco entre agregados } \\
\text { desse volume de solo são nulas, mas a estabilidade e a coesão dos agregados } \\
\text { podem ser altas. A porosidade a olho nu é importante. }\end{array}$ \\
\hline \multirow{3}{*}{$\begin{array}{l}\text { (Somente para } \\
\text { volume AM) }\end{array}$} & $\mathrm{F}$ & $\begin{array}{l}\text { Volume de solo fissurado, em que a individualização de torrões é facilitada pela } \\
\text { fissuração, sendo estes de tamanhos variados. Quando presentes nesse volume, } \\
\text { as raízes se desenvolvem preferencialmente entre os torrões, nas fissuras } \\
\text { existentes. Podem ser bem ramificadas e orientadas em todas as direções, mas } \\
\text { normalmente apresentam aspecto achatado. A porosidade é essencialmente } \\
\text { fissural. }\end{array}$ \\
\hline & Z & $\begin{array}{l}\text { Volume de solo formado essencialmente de estrutura laminar. As raízes, } \\
\text { quando presentes nesse volume, são tortuosas e com desenvolvimento } \\
\text { horizontal. Normalmente, não são ramificadas e, além da tortuosidade, } \\
\text { apresentam aspecto bem achatado. }\end{array}$ \\
\hline & $\mathrm{C}$ & $\begin{array}{l}\text { Volume de solo em que os elementos (agregados e terra fina) estão unidos, } \\
\text { formando um volume bastante homogêneo, com aspecto de estrutura maciça, } \\
\text { sendo impossível a individualização de torrões a olho nu. Pode apresentar } \\
\text { raízes em grandes quantidades, bem ramificadas, não achatadas e não } \\
\text { tortuosas, orientadas em todas as direções, quando o volume não for compacto, } \\
\text { e, ou, não ramificadas, achatadas e tortuosas, orientadas horizontalmente, } \\
\text { quando o volume for compacto. A porosidade é essencialmente de empilhamento } \\
\text { de agregados, podendo apresentar cavidades arredondadas e, ou, poros } \\
\text { tubulares. }\end{array}$ \\
\hline
\end{tabular}

\footnotetext{
(1) Nos modos de organização L e F, além do estado interno dos torrões, devem-se classificar os torrões pelo tamanho: 1 a 5 cm =
} pequenos; 6 a $10 \mathrm{~cm}=$ médios; $>10 \mathrm{~cm}=$ grandes. 


\section{Quadro 2. Estado interno dos torrões presentes nos diferentes modos de organização do volume de solo antropizado}

\begin{tabular}{|c|c|c|}
\hline Nível de análise & Simbologia & Definição \\
\hline \multirow{5}{*}{111} & $\begin{array}{l}\mu \\
\text { (Agregado } \\
\text { não } \\
\text { compacto) }\end{array}$ & $\begin{array}{l}\text { Estado interno dos torrões caracterizado por uma distribuição de agregados } \\
\text { com estrutura interna e externa porosa, fácil de ser observada a olho nu, com } \\
\text { predominância de poros tipo amontoamento de agregados. Normalmente, } \\
\text { apresenta raízes intra e entre agregados, bem ramificadas, não achatadas, com } \\
\text { orientação vertical não prejudicada pela compactação. As faces de ruptura são } \\
\text { rugosas e a coesão a seco é pequena. }\end{array}$ \\
\hline & $\begin{array}{c}\Delta \\
\text { (Agregado } \\
\text { compacto) }\end{array}$ & $\begin{array}{l}\text { Estado interno de torrões compactados, caracterizado por uma distribuição de } \\
\text { agregados com estrutura angulosa (poliédrica, cúbica ou prismática), devido à } \\
\text { forte pressão externa, com uma porosidade visível a olho nu muito pouco } \\
\text { desenvolvida, com predominância, quando existir, de poros tubulares e, ou, } \\
\text { cavidades arredondadas, podendo existir fissuras. Quase não apresenta raízes e } \\
\text { estas, quando presentes, possuem poucas ramificações. São achatadas, com } \\
\text { orientação vertical prejudicada pela compactação. As faces de ruptura são } \\
\text { principalmente lisas e a coesão a seco é muito elevada. }\end{array}$ \\
\hline & & $\begin{array}{l}\text { Estado intermediário entre agregados compactos e não compactos, com duas } \\
\text { possibilidades: }\end{array}$ \\
\hline & $\begin{array}{c}\mu \Delta / \Delta \mu \\
\text { (Agregado } \pm \\
\text { compacto) }\end{array}$ & $\begin{array}{l}\text { (1) Estado }(\mu \Delta) \text { : agregados que estão em processo de compactação, mas que } \\
\text { ainda guardam predominantemente as características do estado não compacto } \mu \\
\text { sobre as características do estado compacto } \Delta \text { (definidos acima); }\end{array}$ \\
\hline & & $\begin{array}{l}\text { (2) Estado }(\Delta \mu) \text { : agregados que estão bem compactos, mas que ainda guardam } \\
\text { algumas características do estado não compacto } \mu \text { (definido acima). }\end{array}$ \\
\hline
\end{tabular}

Obs: Durante a análise do perfil cultural, se forem observados volumes de solo com problema de hidromorfia, volumes com concentração de matéria orgânica ou resultantes de atividade biológica, estes devem ser indicados com a seguinte simbologia: $\mathbf{h}$ para indicar hidromorfia; b para indicar atividade biológica, e mo para indicar matéria orgânica.

(NAM), para, em seguida, descrever cada tipo de organização dos vol umes (AM), com a maior riqueza de detal hes possível. Numa segunda fase (Quadro 2), descreve-se o estado interno dos torrões presentes nos diferentes modos de organização do volume de solo mobilizado (nível II - Quadro 1). Nessa descrição, que deverá ser bastante criteriosa, consideram-se a estrutura dos agregados, a porosidade, a estabilidade em água, a coesão a seco, as faces de ruptura e o enraizamento.

\section{RESULTADOS E CONSIDERAÇÕE S FINAIS}

Por ser o Perfil Cultural um método qualitativo que permite fazer diagnósticos do estado estrutural do sol o no campo, levando em conta a heterogeneidade do meio físico trabalhado, procurou-se estudar alguns efeitos específicos, como o mecanismo de compactação, os efeitos de implementos no solo, dentre outros, em Latossolos argilosos a partir de diferentes manejos de sol os, com o objetivo de testar o método.

Em uma área de Latossolo Roxo cultivada com citros e diferentes adubos verdes, analisou-se a evolução das estruturas após 9 anos de implantação do pomar (Figura 1).
Pode-se observar que o sol o apresenta estruturas tendendo à compactação $(F \Delta \mu, C \Delta \mu)$ e, em alguns pontos, estruturas bem compactas tipo $(C \Delta)$, em comparação com um mesmo solo sob mata natural localizado a menos de cem metros do experimento em questão, onde o solo é poroso com predomínio de estruturas $\mathrm{C} \mu$ por todo o perfil. Isso é devido, provavel mente, ao tráfego de máquinas por ocasião das operações de roçagem da área e colheita da produção.

Dersigny et al. (1990) caracterizaram os diferentes estados estruturais em um L atossol o Roxo cultivado nos sistemas de semeadura convencional e direta, observando particularmente a ocorrência de compactação. A descrição do Perfil Cultural mostrou a presença de camada compactada $(\mathrm{C} \Delta, \mathrm{F} \Delta)$ nos dois sistemas, mas com diferenças em características físicas como profundidade de ocorrência, espessura e densidade. A observação do enraizamento mostrou que as raízes exploravam preferencialmente as estruturas fragmentadas $(F)$ em relação às estruturas maciças (C), podendo esse critério ser preponderante ao fator densidade. A partir disso, uma informação de cunho prático foi relatada por Ral isch et al . (1991), os quais afirmaram que a observação criteriosa da morfologia do solo e das raízes permite mel hor compreensão da interação implemento-solo-planta. 


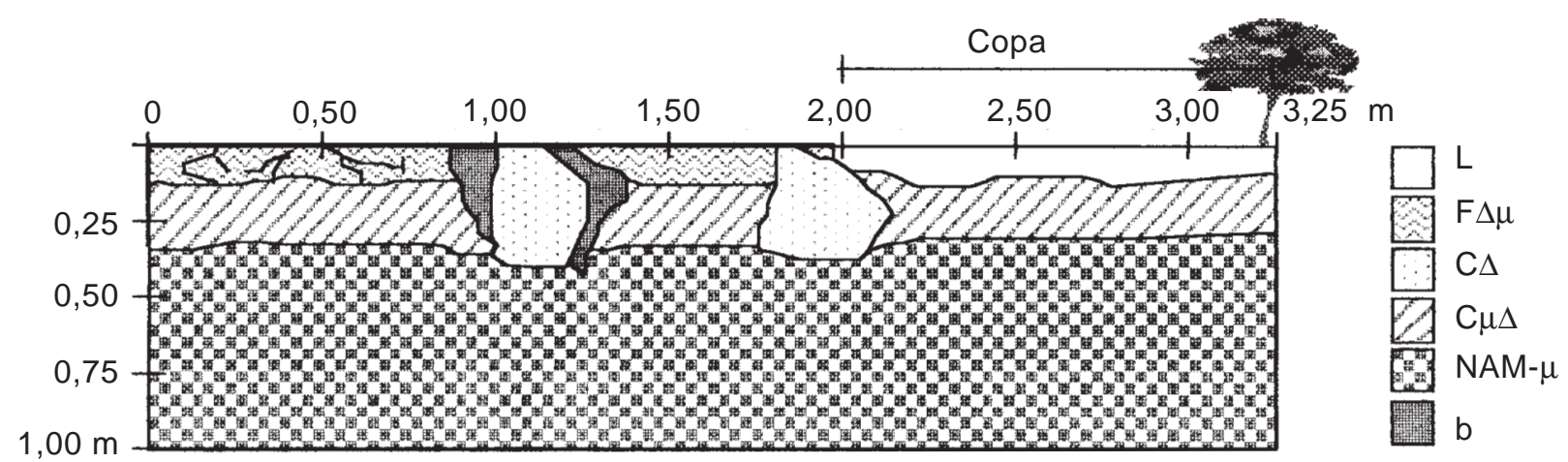

Figura 1. Perfil Cultural sob cultura de citros em LRd (Londrina-PR).

Ralisch et al. (1994) utilizaram o Perfil Cultural para avaliar os efeitos da semeadura direta, do preparo convencional e da gradagem pesada sobre as condições físicas e enraizamento de culturas. Concluíram que o Perfil Cultural tem sua eficiência aumentada quando acompanhado de dados analíticos de amostras retiradas a partir das estruturas observadas no perfil, pois, no caso de camadas $\Delta \mu$ e $\mu \Delta$, por exemplo, os resultados analíticos são um suporte maior para tirar conclusões mais seguras.

Ralisch (1995) comparou a estrutura de um Latossolo submetido a diferentes sistemas de preparo com a de um sob vegetação de floresta de pinus e observou que a semeadura direta ocasionou compactação até $50 \mathrm{~cm}$ com estruturas tipo $\mathrm{C} \mu \Delta$ ou $\mathrm{C} \Delta \mu$, enquanto o sistema de preparo com arado de aivecas proporcionou melhores condições para o enraizamento do trigo, pois houve um predomínio de camadas $\mathrm{F}$, com estruturas $\Delta \mu$.

Tavares Filho (1995) comparou, com a ajuda do método do Perfil Cultural, o comportamento de dois Latossolos, sendo um eutrófico e outro distrófico, submetidos ao sistema de semeadura direta e preparo com arado de aivecas. $\mathrm{O}$ autor demonstrou (Figura 2) que o tipo de preparo que fornece as mel hores condições físicas ao sol o pode ser diferente em função do tipo de solo. Para o solo distrófico, a semeadura direta forneceu as melhores condições de porosidade (da ordem de $60 \%$ ), com predomínio da estrutura contínua - $\mathrm{C} \mu \Delta$. No eutrófico, foi o arado de aivecas que forneceu as melhores condições de porosidade (da ordem de $54 \%$ para o arado de aivecas contra $48 \%$ na semeadura direta), pois houve, neste caso, um predomínio da estrutura com fissuras - $F \mu \Delta$.

Em área de cana-de-açúcar sob Terra Roxa Estruturada, avaliou-se a evolução das estruturas do solo (Figura 3). Observou-se, com o auxílio do Perfil Cultural, quea compactação estava presente, com estruturas principalmente do tipo Z (área de rodado das máquinas), e $C \Delta$ praticamente por todo o perfil. Nos sulcos de plantio, observou-se uma estrutura livre (L), com torrões relativamente grandes $(>10 \mathrm{~cm})$, mais ou menos compactos $(\Delta \mu)$, entre os quais as raízes se desenvolveram preferencialmente. Tais resultados concordam com os de Medina (1993) que, estudando o efeito da aplicação de vinhaça e gesso em cultura de cana-deaçúcar sobre as características do solo, observou que a aplicação de vinhaça pode ocasi onar a compactação, com estruturas principalmente do tipo $\mathrm{C} \Delta$ ou $\mathrm{F} \Delta$, quando ocorreu intenso tráfego de máquinas.
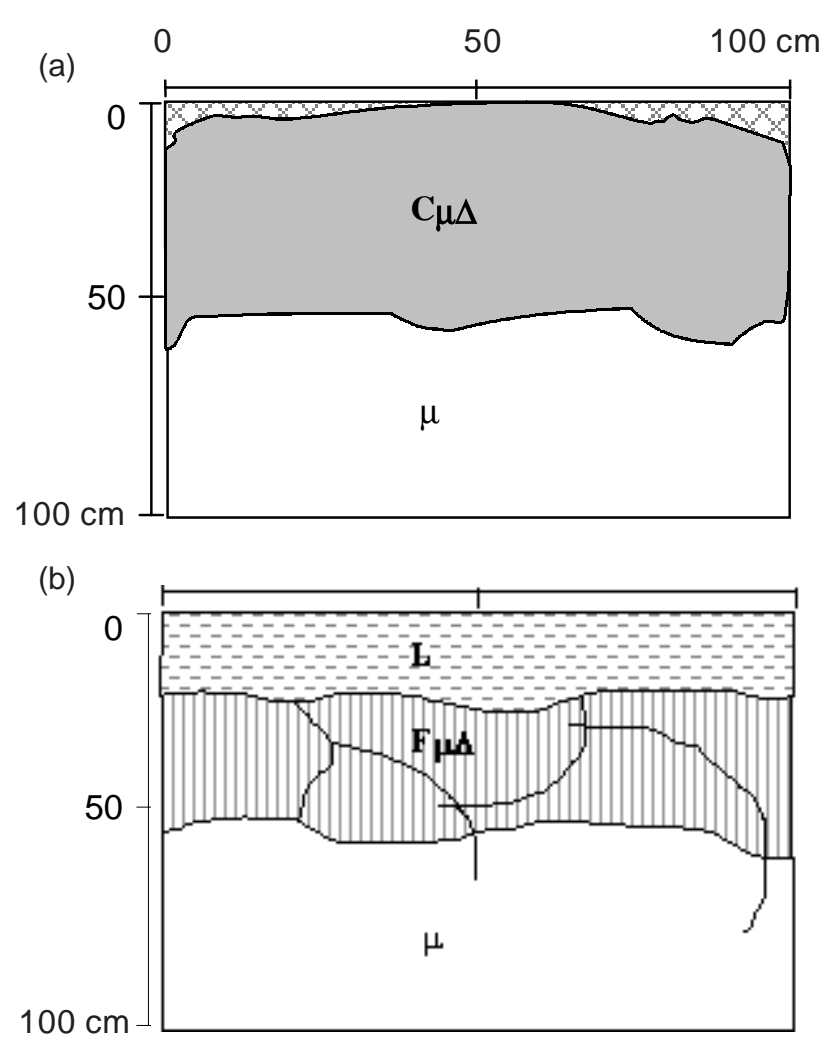

Figura 2. Perfis Culturais: (a) sob semeadura direta em LRd (Cascavel - PR), (b) sob arado de aivecas em LR (Palotina-PR). 


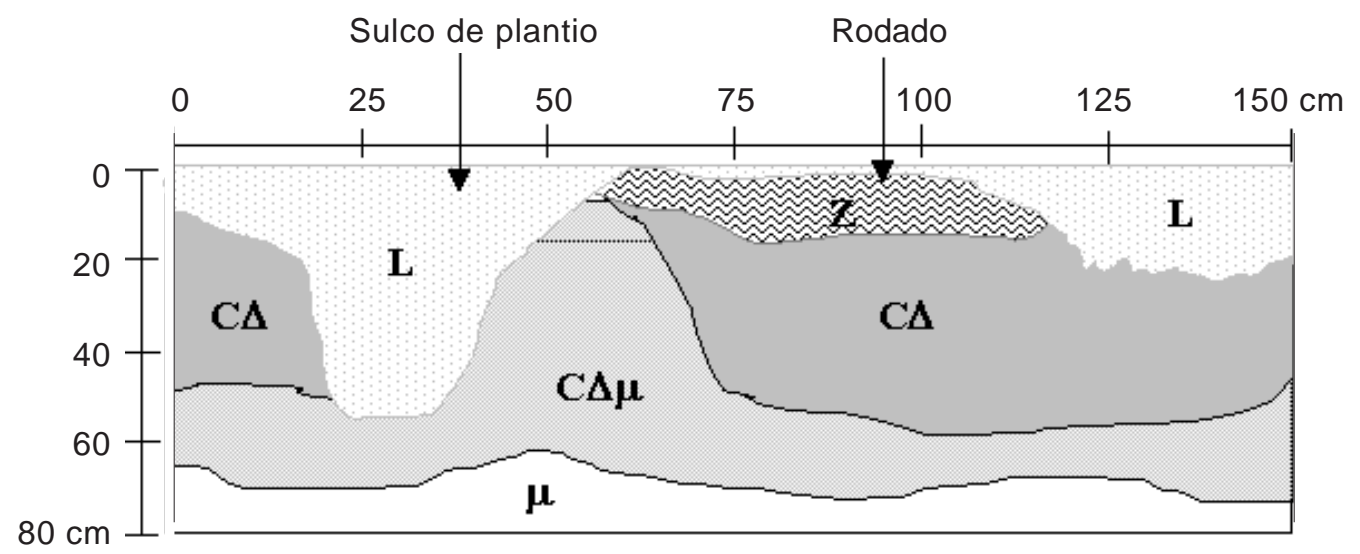

Figura 3. Perfil Cultural sob área de cana-de-açúcar em TRE (Igaraçu do Tietê-SP).

Esse método tem-se mostrado eficiente não só no estudo da variação estrutural, mas também no estudo das interações das características físicas, químicas e biológicas do solo, que são afetadas pela porosidade e movimentação da água. Pelo fato de não se poder considerar somente o estado físico atual do meio, deve-se tentar também prever ou compreender sua história para se chegar a um diagnóstico correto no campo.

Além dessa utilização em diagnósticos, o método oferece outras possibilidades de utilização, a saber:

- orientação na amostragem do solo, levando em consideração suas diferenças espaciais;

- auxílio no estudo dos efeitos de máquinas e implementos agrícolas no solo;

- facilidade na visualização das interações físicas, químicas e biológicas dos solos, e

- meio de auxílio no estudo do sistema radicular.

\section{LITERATURA CITADA}

CHARREAU, C. \& NICOU, R. L'amélioration du profil cultural dans les sols sableux et sablo-argileux de la zone tropicale sèche ouest-africaine et ses incidences agronomiques. Paris, IRAT, 1971. 254p. (Bulletin Agronomique, 23)

DERSIGNY, C.; GUIMARÃES, M.F. \& VISINTIN, T.M.R. Observação do estado estrutural e da repartição espacial do sistema radicular do milho cultivado num Latossolo Roxo. In: CONGRESSO BRASILEIRO E ENCONTRO DE PESQUISA SOBRE CONSERVAÇÃO DE SOLO, 8., Londrina, 1990. Resumos. Londrina, Sociedade Brasileira de Ciência do Solo,1990. p.76.

GAUTRONNEAU, Y.\& MANICHON, H. Guide méthodique du profil cultural. Lyon, CEREF-GEARA, 1987. 71p.

GRAS, R.; OSTY, P.L.; DEFFONTAINES, J.P. \& MARINLAFLÉCHE, A. Contribuition à l'étude de la culture de la betterave à sucre sur des sols légers du Leannois et de la Champagne de L'Aisne. Ann. Agron., 22:537-584, 1971.
GUIMARÃES, M.F.; RALISCH, R. \& MEDINA, C.C. O perfil cultural e as modificações da estrutura do solo. In: CONGRESSO BRASILEIRO DE CIÊNCIA DO SOLO, 24., Goiânia, 1993. Resumos. Goiânia, Sociedade Brasileira de Ciência do Solo, 1993. p.23-24.

HENIN, S.; FEODOROFF, A.; GRAS, R. \& MONNIER, G. Leprofil cultural: Principes de physique du sol. Paris, Masson, 1960. 320p.

HENIN, S.; GRAS, R. \& MONNIER, G. Le profil cultural : L'état physique du sol et ses conséquences agronomiques. 2.ed. Paris, Masson, 1969. 322p.

LETEY, J . Relationship between soil physical properties and crop production. Adv. Soil Sci., 1:277-294, 1985.

MANICHON, H. Influence des systèmes de culture sur le profil cultural: élaboration d'une méthode de diagnostic bassée sur I'observation morphologique. Paris, INA-PG.1982. 241p. (Tese de Doutorado)

MANICHON, H. Compactage, décompactage du sol et systémes de culture. C.R. Acad. Agric., 74:43-54, 1988.

MANICHON, H. \& ROGER ESTRADA, J. Caractérisation de l'état structural et étude de son évolution à court et moyen terme sous l'action des systémes de culture. In: COMBE, L. \& PICARD, D., eds. Les systémes de culture. Paris, INRA, 1990. p.27-55.

MANICHON, H. Le profil cultural : une perspective nouvelle pour I'analyse du travail du sol. In: RÉUNION DU GROUPE THÉMATIQUE: STRUCTURE ET FERTILITÉ DES SOLS TROPICAUX, 2., Montpellier, 1995. Anais. Montpellier, ORSTOM, 1995. p.7-14.

MEDINA, C.C. Estudo da aplicação de gesso, cal cário e vinhaça na produção e enraizamento de cana-de-açúcar (Saccharum spp). Botucatu, Universidade Estadual Paulista, 1993. 175p. (Tese de Doutorado)

PAPY, F. Effet de l'état structural d'une couche labourée sur sa rétention en eau. Agronomie, 6:57-66, 1986.

RALISCH, R. Efeito de três sistemas de manejo no estado estrutural de um Latossolo Roxo. Botucatu, Universidade Estadual Paulista, 1995. 65p. (Tese de Mestrado) 
RALISCH, R.; GUIMARÃES, M.F.; MEDINA, C.C.; TAVARES FILHO, J .; DERSIGNY, C.G. \& VISINTIN, L.M.B. O método do perfil cultural para auxiliar na avaliação dos efeitos do preparo sobre a estrutura do solo. In: CONGRESSO BRASILEIRO DE ENGENHARIA AGRÍCOLA, 20., Londrina, 1991. Anais. Londrina, Sociedade Brasileira de Engenharia Agrícola, 1991. p.1374-1382.

RALISCH, R.; GUIMARÃES, M.F . \& MEDINA, C.C. Thecultural profile methodology utilization to evaluate thetillage effects and tillage equipment efficiency. In: WORLD CONGRESS ON AGRICULTURAL ENGINEERING, 12., Milano, 1994. Proceedings. Merel beke, CI GR, 1994. p.1273-1279.

RALISCH, R.; TAVARES FILHO, J .; GUIMARÃES, M.F.\& MEDINA, C.C. Utilisation du profil cultural pour l'étude de la compaction des sols ferralitiques de l'état du Paraná (Brésil). In: RÉUNION DU GROUPE THÉMATIQUE : STRUCTURE ET FERTILITÉ DES SOLSTROPICAUX, 2., Montpellier, 1995. Anais. Montpellier, ORSTOM, 1995. p.57-62.
TARDIEU, F. \& MANICHON, H. Caractérisation en tant que capteur d'eau de l'enracinement du maïs en parcelle cultivée. Une métode d'étude de la répartition verticale et horizontale des racines. Agronomie, 6:415-425, 1986.

TARDIEU, F. Analysis of spatial variability of maizeroot density. Distances between roots. Plant Soil, 107:259-266, 1988.

TAVARES FILHO, J .; GUIMARÃES, M.F.; RALISCH, R.; MEDINA, C.C.; DERSIGNY, C.G. \& VISINTIN, L.M.B. Utilização do método do perfil cultural para avaliação do desenvolvimento de uma cultura. In: CONGRESSO BRASILEIRO DE CIÊNCIA DO SOLO, 23., Porto Alegre, 1991. Resumos. Porto Alegre, Sociedade Brasileira de Ciência do Solo, 1991. p.142.

TAVARES FILHO, J . Organisation et comportement des Latosols du Paraná (Brésil). Influencedeleur miseen culture. Nancy, Université de Nancy I, 1995. 229p. (Tese de Doutorado) 